\title{
BMC Pulmonary Medicine reviewer acknowledgement 2015
}

Dirk Krüger

\section{Contributing reviewers}

The editors of BMC Pulmonary Medicine would like to thank all our reviewers who have contributed to the journal in Volume 15 (2015).

Alen Faiz

Netherlands

As Hoza

Tanzania

Adam Collison

Australia

Adrian Ceccato

Argentina

Afroditi Boutou

Greece

Michelle Murray

Ireland

Aisling McGowan

Ireland

Akash Verma

Singapore

Nobuhiro Akuzawa

Japan

Albert Lim

Singapore

Anantham Devanand

Singapore

Andras Bikov

Hungary

Andrea Wolfler

Italy
Andreas Hector

Germany

Andrea Zanoni

Italy

Andrew Argent

UK

Anjali Ralhan

Germany

Anna Selvaggio

USA

Annemarie Lee

Australia

Ozsancak Ugurlu

UK

Luis Nannini

Argentina

Carlos Nigro

Argentina

Anand Agrawal

India

Kjetil Ask

Canada

Joerg Mattes

Australia

Paul Thomas

Australia
Narelle Cox
Australia

Uwana Evers

Australia

Chris Del Mar

Australia

Maher Almatar

Australia

Rakesh Kumar

Australia

Dino Bee Aik Tan

Australia

Peter Franklin

Australia

Jodie Simpson

Australia

Bircan Erbas

Australia

Dianne Goeman

Australia

Richard Kim

Australia

Vanessa McDonald

Australia

Andre Schultz

Australia

Correspondence: dirk.krueger@biomedcentral.com

BioMed Central, Floor 6, 236 Gray's Inn Road, London WC1X 8HB, UK 
Biarta Rhys-Jones

Australia

Graeme Zosky

Australia

Christopher Zappala

Australia

Mark Everard

Australia

Leanne Rodwell

Australia

Vicki Clifton

Australia

Vanessa Murphy

Australia

Dennis Lau

Australia

Bronwyn Berthon

Australia

Paul Robinson

Australia

Rosalba Courtney

Australia

Gavin Pinniger

Australia

Jutta Horejs-Hoeck

Austria

Balazs Hegedus

Austria

Antoni Xaubet

Spain

Sandra Baldacci

Italy

Begum Ergan

Italy

Benoit Nemery

Belgium

Yang Bai

Belgium

Nicolas Lanthier

Belgium

Ghislaine Gayan-Ramirez

Belgium

Blanca Himes

USA
Bettina F Loza

Netherlands

Brian McCullagh

Ireland

Eanes Pereira

Brazil

Daniela Andaku

Brazil

Lia Bittencourt

Brazil

Mariana Ferreira

Brazil

Andre Albuquerque

Brazil

Emilio Pizzichini

Brazil

Bruno Bezerril Andrade

Brazil

Bruno Balbi

Italy

Barbara Yawn

USA

Collin Brooks

New Zealand

Craig Winstanley

UK

Luca Cabrini

Italy

Michael Parkins

Canada

Bradley Quon

Canada

Pat Camp

Canada

Michael Stickland

Canada

Gambo Aliyu

Canada

John Garvey

Canada

Dennis Jensen

Canada

Jeremy Road

Canada
Corey Tomczak

Canada

Adam Shapiro

Canada

Adrian West

Canada

David Ngan

Canada

Anthony D'Urzo

Canada

Carlos Aravena

Chile

Carlo Caffarelli

Italy

Carlos Martinez

USA

Carsten Schwarz

Germany

Paolo Ceppi

Italy

Wenjun Mou

China

Nong J Zhang

China

Duo Zhang

China

Mingzhou Guo

China

Yan Jia

China

Tianwen Lai

China

Sun Hongmei

China

Eusebi Chiner

Spain

Davide Chiumello Italy

Christian Becker

USA

Christophe Dooms

Belgium

Christophe Miles

France 


\begin{tabular}{|c|c|c|}
\hline $\begin{array}{l}\text { Gianluca Campo } \\
\text { Italy }\end{array}$ & $\begin{array}{l}\text { Emily Wan } \\
\text { USA }\end{array}$ & $\begin{array}{l}\text { Matthias Griese } \\
\text { Germany }\end{array}$ \\
\hline $\begin{array}{l}\text { John Cockroft } \\
\text { UK }\end{array}$ & $\begin{array}{l}\text { Eduard Monsó } \\
\text { Spain }\end{array}$ & $\begin{array}{l}\text { Saeed Kolahian } \\
\text { Germany }\end{array}$ \\
\hline $\begin{array}{l}\text { Connie Yang } \\
\text { Canada }\end{array}$ & $\begin{array}{l}\text { Erica Rutten } \\
\text { Netherlands }\end{array}$ & $\begin{array}{l}\text { Joachim Riethmüller } \\
\text { Germany }\end{array}$ \\
\hline $\begin{array}{l}\text { Sanja Popovic-Grle } \\
\text { Croatia }\end{array}$ & $\begin{array}{l}\text { Kelemework Adane } \\
\text { Ethiopia }\end{array}$ & $\begin{array}{l}\text { Elaine Fuertes } \\
\text { Germany }\end{array}$ \\
\hline $\begin{array}{l}\text { Davor Plavec } \\
\text { Croatia }\end{array}$ & $\begin{array}{l}\text { Eric Wong } \\
\text { Canada }\end{array}$ & $\begin{array}{l}\text { Melanie Königshoff } \\
\text { Germany }\end{array}$ \\
\hline $\begin{array}{l}\text { Christina Tischer } \\
\text { Germany }\end{array}$ & $\begin{array}{l}\text { Fabiano Di Marco } \\
\text { Italy }\end{array}$ & $\begin{array}{l}\text { Helge Hebestreit } \\
\text { Germany }\end{array}$ \\
\hline $\begin{array}{l}\text { Vitezslav Kolek } \\
\text { Czech Republic }\end{array}$ & $\begin{array}{l}\text { Felip Burgos } \\
\text { Spain }\end{array}$ & $\begin{array}{l}\text { Johannes Bickenbach } \\
\text { Germany }\end{array}$ \\
\hline $\begin{array}{l}\text { Olga Krystufkova } \\
\text { Czech Republic }\end{array}$ & $\begin{array}{l}\text { Olli Ruuskanen } \\
\text { Finland }\end{array}$ & $\begin{array}{l}\text { Alexander Kersten } \\
\text { Germany }\end{array}$ \\
\hline $\begin{array}{l}\text { Martina Vasakova } \\
\text { Czech Republic }\end{array}$ & $\begin{array}{l}\text { Francesco Puma } \\
\text { Italy }\end{array}$ & $\begin{array}{l}\text { Jan Spillner } \\
\text { Germany }\end{array}$ \\
\hline $\begin{array}{l}\text { Martina Sterclova } \\
\text { Czech Republic }\end{array}$ & $\begin{array}{l}\text { Raphael Borie } \\
\text { France }\end{array}$ & $\begin{array}{l}\text { Nils von Neuhoff } \\
\text { Germany }\end{array}$ \\
\hline $\begin{array}{l}\text { Lukas Capek } \\
\text { Czech Republic }\end{array}$ & $\begin{array}{l}\text { Fanny Rancière } \\
\text { France }\end{array}$ & $\begin{array}{l}\text { Erich Stoelben } \\
\text { Germany }\end{array}$ \\
\hline $\begin{array}{l}\text { David Bennett } \\
\text { Italy }\end{array}$ & $\begin{array}{l}\text { Amelie Guihot } \\
\text { France }\end{array}$ & $\begin{array}{l}\text { Johanna Luca Leinert } \\
\text { Germany }\end{array}$ \\
\hline $\begin{array}{l}\text { Deborah Assayag } \\
\text { Canada }\end{array}$ & $\begin{array}{l}\text { Sylvie Boisramé } \\
\text { France }\end{array}$ & $\begin{array}{l}\text { Joachim Müller-Quernheim } \\
\text { Germany }\end{array}$ \\
\hline $\begin{array}{l}\text { Runa Vavia Fenger } \\
\text { Denmark }\end{array}$ & $\begin{array}{l}\text { Stanislas Ledochowski } \\
\text { France }\end{array}$ & $\begin{array}{l}\text { Michael Kreuter } \\
\text { Germany }\end{array}$ \\
\hline $\begin{array}{l}\text { Dilip Shah } \\
\text { USA }\end{array}$ & $\begin{array}{l}\text { Sebastien Chanoine } \\
\text { France }\end{array}$ & $\begin{array}{l}\text { Nikolaus Becker } \\
\text { Germany }\end{array}$ \\
\hline $\begin{array}{l}\text { A. Daniel Martin } \\
\text { USA }\end{array}$ & $\begin{array}{l}\text { Bernard Maitre } \\
\text { France }\end{array}$ & $\begin{array}{l}\text { Jan Hendrik Storre } \\
\text { Germany }\end{array}$ \\
\hline $\begin{array}{l}\text { Alarico Ariani } \\
\text { Italy }\end{array}$ & $\begin{array}{l}\text { Bruno Crestani } \\
\text { France }\end{array}$ & $\begin{array}{l}\text { Thomas Karlas } \\
\text { Germany }\end{array}$ \\
\hline $\begin{array}{l}\text { Deepa Rastogi } \\
\text { USA }\end{array}$ & $\begin{array}{l}\text { Francesco Bonella } \\
\text { Germany }\end{array}$ & $\begin{array}{l}\text { Florian Fuchs } \\
\text { Germany }\end{array}$ \\
\hline $\begin{array}{l}\text { Elżbieta Radzikowska } \\
\text { Poland }\end{array}$ & $\begin{array}{l}\text { Ertunc Altiok } \\
\text { Germany }\end{array}$ & $\begin{array}{l}\text { Peter Kardos } \\
\text { Germany }\end{array}$ \\
\hline $\begin{array}{l}\text { Edurne Almirall } \\
\text { Spain }\end{array}$ & $\begin{array}{l}\text { Christian G Cornelissen } \\
\text { Germany }\end{array}$ & $\begin{array}{l}\text { Heinrich Worth } \\
\text { Germany }\end{array}$ \\
\hline $\begin{array}{l}\text { Elena Gimeno-Santos } \\
\text { Spain }\end{array}$ & $\begin{array}{l}\text { Gernot Zissel } \\
\text { Germany }\end{array}$ & $\begin{array}{l}\text { Dominik Hartl } \\
\text { Germany }\end{array}$ \\
\hline $\begin{array}{l}\text { Eliecer Coto } \\
\text { Spain }\end{array}$ & $\begin{array}{l}\text { Nico Derichs } \\
\text { Germany }\end{array}$ & $\begin{array}{l}\text { Peter Gilligan } \\
\text { USA }\end{array}$ \\
\hline
\end{tabular}




\begin{tabular}{|c|c|c|}
\hline $\begin{array}{l}\text { Giuseppe Verlato } \\
\text { Italy }\end{array}$ & $\begin{array}{l}\text { Mehrnoosh Doroudchi } \\
\text { Iran }\end{array}$ & $\begin{array}{l}\text { Maddalena Giannella } \\
\text { Italy }\end{array}$ \\
\hline $\begin{array}{l}\text { Theodore Dassios } \\
\text { Greece }\end{array}$ & $\begin{array}{l}\text { Liam Cormican } \\
\text { Ireland }\end{array}$ & $\begin{array}{l}\text { Simone Dore } \\
\text { Italy }\end{array}$ \\
\hline $\begin{array}{l}\text { Christina Kalogeropoulou } \\
\text { Greece }\end{array}$ & $\begin{array}{l}\text { Shane O'Neill } \\
\text { Ireland }\end{array}$ & $\begin{array}{l}\text { Cristiano Fava } \\
\text { Italy }\end{array}$ \\
\hline $\begin{array}{l}\text { Sophia Schiza } \\
\text { Greece }\end{array}$ & $\begin{array}{l}\text { Limy Wong } \\
\text { Ireland }\end{array}$ & $\begin{array}{l}\text { Claudia Crimi } \\
\text { Italy }\end{array}$ \\
\hline $\begin{array}{l}\text { Izolde Bouloukaki } \\
\text { Greece }\end{array}$ & $\begin{array}{l}\text { Anne Costello } \\
\text { Ireland }\end{array}$ & $\begin{array}{l}\text { Monica Rocco } \\
\text { Italy }\end{array}$ \\
\hline $\begin{array}{l}\text { Spyros Papiris } \\
\text { Greece }\end{array}$ & $\begin{array}{l}\text { Kirsten Schaffer } \\
\text { Ireland }\end{array}$ & $\begin{array}{l}\text { Salvatore Cazzato } \\
\text { Italy }\end{array}$ \\
\hline $\begin{array}{l}\text { Eirini Roumpedaki } \\
\text { Greece }\end{array}$ & $\begin{array}{l}\text { Donald Silverberg } \\
\text { Israel }\end{array}$ & $\begin{array}{l}\text { Michele Mondoni } \\
\text { Italy }\end{array}$ \\
\hline $\begin{array}{l}\text { Giovanni Sotgiu } \\
\text { Italy }\end{array}$ & $\begin{array}{l}\text { Oleg Dolkart } \\
\text { Israel }\end{array}$ & $\begin{array}{l}\text { Paola De Filippi } \\
\text { Italy }\end{array}$ \\
\hline $\begin{array}{l}\text { Nabeel Hamzeh } \\
\text { USA }\end{array}$ & $\begin{array}{l}\text { Antonello Nicolini } \\
\text { Italy }\end{array}$ & $\begin{array}{l}\text { Paolo Scanagatta } \\
\text { Italy }\end{array}$ \\
\hline $\begin{array}{l}\text { Hans Jörg Baumann } \\
\text { Germany }\end{array}$ & $\begin{array}{l}\text { Davide Colombi } \\
\text { Italy }\end{array}$ & $\begin{array}{l}\text { Mario Olivieri } \\
\text { Italy }\end{array}$ \\
\hline $\begin{array}{l}\text { Helena Backman } \\
\text { Sweden }\end{array}$ & $\begin{array}{l}\text { Cristiano Rampinelli } \\
\text { Italy }\end{array}$ & $\begin{array}{l}\text { Giulio Rossi } \\
\text { Italy }\end{array}$ \\
\hline $\begin{array}{l}\text { Herbert Schiller } \\
\text { Germany }\end{array}$ & $\begin{array}{l}\text { Leonello Fuso } \\
\text { Italy }\end{array}$ & $\begin{array}{l}\text { Annunziata Faustini } \\
\text { Italy }\end{array}$ \\
\hline $\begin{array}{l}\text { Hui Fang Lim } \\
\text { Singapore }\end{array}$ & $\begin{array}{l}\text { Francesco Pistelli } \\
\text { Italy }\end{array}$ & $\begin{array}{l}\text { Raffaele Antonelli-Incalzi } \\
\text { Italy }\end{array}$ \\
\hline $\begin{array}{l}\text { Veronika Müller } \\
\text { Hungary }\end{array}$ & $\begin{array}{l}\text { Claudia Ravaglia } \\
\text { Italy }\end{array}$ & $\begin{array}{l}\text { Alessandro Marcon } \\
\text { Italy }\end{array}$ \\
\hline $\begin{array}{l}\text { Jozsef Lovey } \\
\text { Hungary }\end{array}$ & $\begin{array}{l}\text { Dina Visca } \\
\text { Italy }\end{array}$ & $\begin{array}{l}\text { Luca Ampollini } \\
\text { Italy }\end{array}$ \\
\hline $\begin{array}{l}\text { Ian Sayers } \\
\text { UK }\end{array}$ & $\begin{array}{l}\text { Giovanni Volpicelli } \\
\text { Italy }\end{array}$ & $\begin{array}{l}\text { Alessio Rungatscher } \\
\text { Italy }\end{array}$ \\
\hline $\begin{array}{l}\text { Magnus Gottfredsson } \\
\text { Iceland }\end{array}$ & $\begin{array}{l}\text { Fabrizio Luppi } \\
\text { Italy }\end{array}$ & $\begin{array}{l}\text { Cinzia Maspero } \\
\text { Italy }\end{array}$ \\
\hline $\begin{array}{l}\text { Joyashree Banerjee } \\
\text { India }\end{array}$ & $\begin{array}{l}\text { Silvano Gallus } \\
\text { Italy }\end{array}$ & $\begin{array}{l}\text { Cristina Cigana } \\
\text { Italy }\end{array}$ \\
\hline $\begin{array}{l}\text { Anurag Agrawal } \\
\text { India }\end{array}$ & $\begin{array}{l}\text { Lara Pisani } \\
\text { Italy }\end{array}$ & $\begin{array}{l}\text { Nicola Ciancio } \\
\text { Italy }\end{array}$ \\
\hline $\begin{array}{l}\text { Arjun Ram } \\
\text { India }\end{array}$ & $\begin{array}{l}\text { Marco Confalonieri } \\
\text { Italy }\end{array}$ & $\begin{array}{l}\text { Federico Lavorini } \\
\text { Italy }\end{array}$ \\
\hline $\begin{array}{l}\text { Susi Ari Kristina } \\
\text { Indonesia }\end{array}$ & $\begin{array}{l}\text { Claudio Pedone } \\
\text { Italy }\end{array}$ & $\begin{array}{l}\text { Mario Mascalchi } \\
\text { Italy }\end{array}$ \\
\hline $\begin{array}{l}\text { Indre Butiene } \\
\text { Lithuania }\end{array}$ & $\begin{array}{l}\text { Marco Falcone } \\
\text { Italy }\end{array}$ & $\begin{array}{l}\text { Annalisa Carlucci } \\
\text { Italy }\end{array}$ \\
\hline
\end{tabular}




\begin{tabular}{|c|c|c|}
\hline Guido Vagheggini & Masanori Kitaichi & Stefan Krüger \\
\hline Italy & Japan & UK \\
\hline $\begin{array}{l}\text { Francesco Fanfulla } \\
\text { Italy }\end{array}$ & $\begin{array}{l}\text { Takeshi Kawasaki } \\
\text { Japan }\end{array}$ & $\begin{array}{l}\text { Laura Crotty Alexander } \\
\text { USA }\end{array}$ \\
\hline $\begin{array}{l}\text { Carmelo Caserta } \\
\text { Italy }\end{array}$ & $\begin{array}{l}\text { Takeshi Johkoh } \\
\text { Japan }\end{array}$ & $\begin{array}{l}\text { Chung-Tat Lun } \\
\text { Hong Kong }\end{array}$ \\
\hline $\begin{array}{l}\text { Mario Cazzola } \\
\text { Italy }\end{array}$ & $\begin{array}{l}\text { Atsushi Nambu } \\
\text { Japan }\end{array}$ & $\begin{array}{l}\text { Eleftherios Zervas } \\
\text { Greece }\end{array}$ \\
\hline $\begin{array}{l}\text { Susanna Esposito } \\
\text { Italy }\end{array}$ & $\begin{array}{l}\text { Nobuhiko Nagata } \\
\text { Japan }\end{array}$ & $\begin{array}{l}\text { Lenka Munoz } \\
\text { Australia }\end{array}$ \\
\hline $\begin{array}{l}\text { Iraklis Tsangaris } \\
\text { Greece }\end{array}$ & $\begin{array}{l}\text { Jeffrey Horowitz } \\
\text { USA }\end{array}$ & $\begin{array}{l}\text { Leslie Grammer } \\
\text { USA }\end{array}$ \\
\hline $\begin{array}{l}\text { Isabelle Vivodtzev } \\
\text { France }\end{array}$ & $\begin{array}{l}\text { Joao Winck } \\
\text { UK }\end{array}$ & $\begin{array}{l}\text { Kathleen Lindell } \\
\text { USA }\end{array}$ \\
\hline $\begin{array}{l}\text { Joanna Chorostowska-Wynimko } \\
\text { Poland }\end{array}$ & $\begin{array}{l}\text { Javier Diaz-Mendoza } \\
\text { USA }\end{array}$ & $\begin{array}{l}\text { Lokesh Guglani } \\
\text { USA }\end{array}$ \\
\hline $\begin{array}{l}\text { Hans Daniels } \\
\text { Netherlands }\end{array}$ & $\begin{array}{l}\text { Jean-Francois Mornex } \\
\text { France }\end{array}$ & $\begin{array}{l}\text { Louisa Owens } \\
\text { Australia }\end{array}$ \\
\hline $\begin{array}{l}\text { Jacques Pralong } \\
\text { Swaziland }\end{array}$ & $\begin{array}{l}\text { Jessica Kasza } \\
\text { Australia }\end{array}$ & $\begin{array}{l}\text { Martin Kneyber } \\
\text { Netherlands }\end{array}$ \\
\hline $\begin{array}{l}\text { Janet Williams } \\
\text { Australia }\end{array}$ & $\begin{array}{l}\text { Jose Herazo-Maya } \\
\text { USA }\end{array}$ & $\begin{array}{l}\text { Massimo Corradi } \\
\text { Italy }\end{array}$ \\
\hline $\begin{array}{l}\text { Yoshinobu Eishi } \\
\text { Japan }\end{array}$ & $\begin{array}{l}\text { Joseph Keane } \\
\text { Ireland }\end{array}$ & $\begin{array}{l}\text { Mark Hew } \\
\text { Australia }\end{array}$ \\
\hline $\begin{array}{l}\text { Shinji Sasada } \\
\text { Japan }\end{array}$ & $\begin{array}{l}\text { Konstantinos Samitas } \\
\text { Greece }\end{array}$ & $\begin{array}{l}\text { Michael Marcus } \\
\text { UK }\end{array}$ \\
\hline $\begin{array}{l}\text { Toru Hifumi } \\
\text { Japan }\end{array}$ & $\begin{array}{l}\text { Karin Valkenet } \\
\text { Netherlands }\end{array}$ & $\begin{array}{l}\text { Marlies Wijseenbeck } \\
\text { Netherlands }\end{array}$ \\
\hline $\begin{array}{l}\text { Shinji Abe } \\
\text { Japan }\end{array}$ & $\begin{array}{l}\text { Barbara Kahl } \\
\text { Germany }\end{array}$ & $\begin{array}{l}\text { Jordan Minov } \\
\text { Macedonia }\end{array}$ \\
\hline $\begin{array}{l}\text { Takushi Namba } \\
\text { Japan }\end{array}$ & $\begin{array}{l}\text { Kumiko Kanatani } \\
\text { Japan }\end{array}$ & $\begin{array}{l}\text { Marcin Zielinski } \\
\text { Poland }\end{array}$ \\
\hline $\begin{array}{l}\text { Takashi Motegi } \\
\text { Japan }\end{array}$ & $\begin{array}{l}\text { Daniel Kass } \\
\text { USA }\end{array}$ & $\begin{array}{l}\text { Mariano Mazzei } \\
\text { Argentina }\end{array}$ \\
\hline $\begin{array}{l}\text { Akira Koarai } \\
\text { Japan }\end{array}$ & $\begin{array}{l}\text { Karen Bernard } \\
\text { USA }\end{array}$ & $\begin{array}{l}\text { Maria Molina-Molina } \\
\text { Spain }\end{array}$ \\
\hline $\begin{array}{l}\text { Mizuho Nishio } \\
\text { Japan }\end{array}$ & $\begin{array}{l}\text { Kevin Pethe } \\
\text { Singapore }\end{array}$ & $\begin{array}{l}\text { Maria Rosaria Bonsignore } \\
\text { Italy }\end{array}$ \\
\hline $\begin{array}{l}\text { Masayuki Hojo } \\
\text { Japan }\end{array}$ & $\begin{array}{l}\text { Seong Yong Lim } \\
\text { South Korea }\end{array}$ & $\begin{array}{l}\text { Martin Andersson } \\
\text { Sweden }\end{array}$ \\
\hline $\begin{array}{l}\text { Atsushi Sano } \\
\text { Japan }\end{array}$ & $\begin{array}{l}\text { Heedoo Lee } \\
\text { South Korea }\end{array}$ & $\begin{array}{l}\text { Masahide Oki } \\
\text { Japan }\end{array}$ \\
\hline $\begin{array}{l}\text { Masashi Bando } \\
\text { Japan }\end{array}$ & $\begin{array}{l}\text { Gi-Byoung Nam } \\
\text { South Korea }\end{array}$ & $\begin{array}{l}\text { Stamatoula Tsikrika } \\
\text { Greece }\end{array}$ \\
\hline
\end{tabular}


Mazen Al-Alawi

Ireland

Michael Dreher

Germany

Megan Jensen

Australia

Jorge Ivan Gamez-Nava

Mexico

Luis Teran

Mexico

Michael Abramson

Australia

Michael Hogardt

Germany

Michal Shteinberg

UK

Claudio Micheletto

Italy

Mark Jennings

USA

Mohamed Manji

Tanzania

Alberto García-Basteiro

Mozambique

Monica Goldklang

USA

Nicholas Glanville

UK

Noelia Cubero de Frutos

Spain

A Knoops

Netherlands

Ivo Wiertz

Netherlands

Pieter Hiemstra

Netherlands

Mark Koelemay

Netherlands

Job van der Palen

Netherlands

Neuza Silva

Portugal

Nicola Murgia

Sweden
Nicola Sverzellati

Italy

Nicolas Girard

France

Nigel Scawn

UK

Ademola Fawibe

Nigeria

Niranjan Jeganathan

USA

Jo Roislien

Norway

Martin Gerdes

Norway

Monica Linea Vold

Norway

Tehmina Mustafa

Norway

Inger Schou Bredal

Norway

Cecilie Svanes

Norway

Ole-Gunnar Anfinsen

Norway

Pankaj Bhavsar

UK

Nousheen Pradhan

Pakistan

Peter Herbison

New Zealand

Pierre Goussard

South Africa

Brian Pickering

UK

Pietro Pirina

Italy

Wieslawa Duszynska

Poland

Magdalena Muc

Poland

Janos Porszasz

USA

Tiago Jacinto

Portugal
Joao Fonseca

Portugal

Yu Li

China

Robert Whiley

UK

Rajesh Bhagat

USA

Rachel Evans

UK

Roberto Santa Cruz

UK

Ritesh Agarwal

India

Ryang Hwa Lee

USA

Rajiv Machado

UK

Robert Siebers

New Zealand

Kari Roberts

UK

Roland Leung

Hong Kong

Stephen Ruoss

USA

Vsevolod Kuzkov

Russia

Shawn Aaron

Canada

Samantha Aso

Spain

Mohamed Regal

Saudi Arabia

Sanjay Chotirmall

Singapore

Souheil El-Chemaly USA

Sergey Dikalov

USA

Sergio Campainha

Portugal

Szymon Skoczynski

Poland 
Yick Hou Lim

Singapore

David Price

Singapore

Sanjay Haresh Chotirmall

Singapore

Simon Walsh

UK

Simon Podnar

Slovenia

Ivan Solovic

Slovakia

Greg Calligaro

South Africa

Keertan Dheda

South Africa

Felipe Cardenal

Spain

Ricard Ramos

Spain

Antonio Esquinas

Spain

Maria Jesus Garcia

Spain

Ernest Nadal

Spain

Jose L Vercher Conejero

Spain

Catia Cilloniz

Spain

Irene Latorre

Spain

Esther Garcia Gil

Spain

Salut Santos

Spain

Stefano Baglioni

Italy

Stefano Sestini

Italy

Stylianos Vittorakis

Greece

Stephan Rosenkranz

Germany
Stephanie Christenson

USA

Stephen Thielke

USA

Sureshbabu Angara

USA

Ann Lindberg

Sweden

Cecilia Stålsby Lundborg

Sweden

Linnea Hedman
Sweden

Jenny Hallberg

Sweden

Konrad Bloch

Switzerland

Wolfgang Jungraithmayr

Switzerland

Werner C Albrich

Switzerland

Sebastian R Ott

Switzerland

Suzanne Cloonan

USA

Toshiaki Jibiki

UK

Tomas Carroll

Ireland

Teck Boon Low

Singapore

Pongdhep Theerawit

Thailand

Rabindra Tirouvanziam

USA

Terumi Midoro-Horiuti

USA

Tony Velkov

Australia

Toshinori Takada

Japan

Guniz Meyanci Koksal

Turkey

Baykal Tulek

Turkey
Hasan Bayram

Turkey

Deus Lukoye

Uganda

Rashid Nadeem

United Arab Emirates

Iram Haq

UK

Kenneth Macleod

UK

Sally Singh

UK

Andrew Wilson

UK

Adrian Martineau

UK

Jennifer Quint

UK

Shoaib Faruqi

UK

Ronan Breen

UK

Timothy Hinks

UK

Daniel Bratton

UK

Helen Chapel

UK

Dhruv Parekh

UK

Ghazwan Butrous

UK

Peter Callery

UK

Andrew Fall

UK

David Spencer

UK

Rachel Jordan

UK

Andrew Bush

UK

Michael Hughes

UK 
Prasad Nagakumar

UK

Xabier Cortés-Lavaud

UK

Francis Gilchrist

UK

Simon Langton Hewer

UK

Roma Maguire

UK

Thomas Wiliams

UK

Eduardo Fernandez Moya

UK

Claire Hogg

UK

Sooky Lum

UK

Neil Thomson

UK

Clive Page

UK

Jonathan Cohen

UK

Gary Latchford

UK

Jens Madsen

UK

Robert Guzy

USA

Julie Ledford

USA

Douglas Curran-Everett

USA

Cory Hogaboam

USA

Jiwang Chen

USA

Haiyang Tang

USA

Pui-Ying Iroh Tam

USA

Stacey Martiniano

USA
Anurag Bajaj

USA

Carolyn Calfee

USA

Stephen Chan

USA

Ying Sun

USA

Peter Moschovis

USA

Hitendra Chand

USA

Venkateshwar Mutyam

USA

Maor Sauler

USA

Girish Nair

USA

Jose Gomez

USA

Rabindra Tirouvanziam

USA

Natasha Williams

USA

Forest Arnold

USA

Deepa Rastogi

USA

Kameswara Rao Badri

USA

Dong-Mei Wang

USA

Jonathan Kropski

USA

Surya Bhatt

USA

Raksha Jain

USA

Deog Kyeom Kim

USA

Bo Shen

USA

Michael Gentile

USA
Samir Kelada

USA

Emily Wan

USA

Khalil Bdeir

USA

Brian Graham

USA

Talat Islam

USA

Jules Lin

USA

Stephen Aronoff

USA

John Ferguson

USA

Rama Mallampalli

USA

Neil Alexis

USA

Amit Gaggar

USA

Timothy Weaver

USA

William Gerthoffer

USA

Matthew Foster

USA

Steve Georas

USA

Karen Bernard

USA

Louise Hecker

USA

Behrouz Jafari

USA

Jessica Bon

USA

Yashoda Hosakote

USA

Tejaswini Kulkarni USA

Sachin Gupte

USA 
Jennifer Trittmann

USA

Suzanne M Cloonan

USA

Praveen Mannam

USA

Edward Miranda

USA

Minfeng Shu

USA

Chao-Hung Lee

USA

Anna Kurdowska

USA

Angela Rogers

USA

Carol Conrad

USA

Ashish Sharma

USA

Asli Gorek Dilektasli

USA

Anand Iyer

USA

Mehdi Mirsaeidi

USA
Kenneth Berger

USA

Janos Porszasz

USA

Trisha Parekh

USA

Hernando Gomez

USA

Philip Westgate

USA

Gary M Hunninghake

USA

Imre Noth

USA

Benjamin Kopp

USA

Nektarios Barabutis

USA

Ming-Hui Fan

USA

Cherry Wongtrakool

USA

Vineet Bhandari

USA

Joseph Swanson

USA
Harikrishnan Parameswaran

USA

Parag Desai

USA

Barry Shea

USA

Vanessa Garcia-Larsen

UK

Victor Kim

USA

Venkateshwar Mutyam

USA

Dong-Mei Wang

USA

Xavier Solanich

Spain

Xue Li Guan

Singapore

Zachary Sellers

USA

Zsolt Pápai-Szekely

Hungary

Zhiyu Dai

USA 